\title{
Review of: "Measuring customer satisfaction on the cleanliness of food premises using fuzzy conjoint analysis: A pilot test"
}

Diego Castro Fettermann

Potential competing interests: The author(s) declared that no potential competing interests exist.

It is an interesting paper which uses fuzzy CA analysis . 